\title{
Öffnet die Schulen!
}

\section{Zur Abwägung der Auswirkungen von Viren und Lockdowns auf Kinder}

\author{
Korrespondenzadresse \\ Prof. Dr. Dr. Manfred Spitzer \\ Universität Ulm \\ Leimgrubenweg 12-14 \\ 87054 Ulm, Deutschland
}

\author{
Bibliografie \\ DOI https://doi.org/10.1055/a-1298-1004 \\ Nervenheilkunde 2021; 40: 296-311 \\ (c) 2021. Thieme. All rights reserved. \\ Georg Thieme Verlag KG, Rüdigerstraße 14, \\ 70469 Stuttgart, Germany \\ ISSN 0722-1541
}

Zusammenfassung: Diese Übersicht wägt das Risiko einer Infektion mit SARS-CoV-2 gegen die Nebenwirkungen von Schulschließungen auf die physische und psychische Gesundheit, auf die Bildung und auf das Wohlbefinden der betroffenen Kinder und Jugendlichen ab. Während kurzfristige Auswirkungen - vermindertes Lernen und geringere Ernährungssicherheit sowie erhöhte Angst, Gewalt gegen Kinder, Kinderarbeit und Teenagerschwangerschaften - häufig Gegenstand der aktuellen Diskussion sind, so werden die langfristigen Auswirkungen von Schulschließungen über die gesamte Lebensspanne der „Generation Corona“ kaum erörtert: Bestehende Pandemien von Inaktivität, Kurzsichtigkeit und Depressionen, von denen bereits Milliarden Menschen betroffen sind, verschlimmern sich durch weniger körperliche Bewegung und weniger Zeit im Freien, schlechte Ernährung, Gewichtszunahme, erhöhte Bildschirmzeit und mehr Einsamkeit während der Schulschließungen, was zu einem zukünftigen Anstieg von Schlaganfall, Herzinfarkt, Krebs und Blindheit führt. Zu den sozio-emotionalen Komplikationen von Isolation und Arbeitslosigkeit gehören erlernte Hilflosigkeit, wirtschaftliche existenzielle Unsicherheit und zunehmende Einsamkeit, was sowohl zu einer Verminderung von Mitgefühl führt als auch das Auftreten von Angststörungen und Depressionen (bis hin zur Suizidalität) begünstigt. Zusammen mit der Verminderung von erreichtem Bildungsstand und wirtschaftlicher Produktivität rechtfertigt das Ausmaß der hierdurch nochmals erhöhten zukünftigen globalen Morbidität und Mortalität die Schließung von Bildungseinrichtungen nur als Ultima Ratio und gebietet deren Öffnung als erste Maßnahme jedweder Lockerung.

Im April 2020 waren von den Schulschließungen während der anhaltenden Corona-Pandemie 1,6 Milliarden Schüler in 190 Ländern betroffen [120]. Während Fabriken und Baustellen brummten, Supermärkte, Apotheken und Drogerien geöffnet waren und deutsche Touristen ins nahe und ferne Ausland reisten (und teilweise mit mutierten Viren zurückkehrten), wurden Kitas und Schulen im Frühjahr 2020 und dann erneut später im Jahr 2020/2021 geschlossen ( $\triangleright$ Abb. 1).

Mit Stand vom 16. März 2021 waren mehr als 120 Millionen Menschen infiziert, mehr als 2,6 Millionen waren gestorben. Neue gefährlichere Mutanten des Erregers SARS-CoV-2 tauchten zunächst in Großbritannien, den USA, Südafrika und Südamerika auf und sind mittlerweile auch bei uns längst angekommen, wo sie das Infektionsgeschehen mit exponentiellem Wachstum erneut beschleunigen. Angesichts der Tatsache, dass Deutschland über weite Strecken dieser Pandemie zu den 10 am stärksten betroffenen Ländern gehörte und die bereits Ende letzten Jahres begonnenen Impfungen bis Ende März nur sehr schleppend voran kamen, blieben Kitas und Schulen über viele Wochen hinweg geschlossen. Sie werden nun wieder geöffnet und möglicherweise bald wieder geschlossen. Ist das tatsächlich „alternativlos“?

Zunächst einmal war und ist die Lage in der Tat ernst. Deutschland befindet sich in der „Dritten Welle“, und es drohen erneut hohe Infektionszahlen mit entsprechend steigender Mortalität. Schließungsmaßnahmen sind wirksam, wenn sie stringent genug umgesetzt werden. Das zeigt nicht nur der Verlauf der Pandemie in China, sondern auch in Israel, Neuseeland und Australien. Israel, das Anfang 2021 im Hinblick auf die Infektionen pro Kopf weltweit am stärksten von SARS-CoV-2 betroffen war, bekämpfte seit dem 8. Januar 2021 die dritte Corona-Welle mit dem dritten „harten“ Lockdown ( $\triangleright$ Abb. 2).

Ein größeres Autorenteam untersuchte kürzlich die Wirksamkeit verschiedener, von Regierungen veranlasster Lockdown-Maßnahmen zur Eindämmung der Corona-Pandemie in 41 Ländern, Schulschließungen [8] erwiesen sich hierbei als vergleichsweise wirksame Maßnahmen ( $\triangleright$ Tab. 1). Ungeklärt ist jedoch die Frage, ob der Effekt tatsächlich auf die nicht mehr über die Schulkinder verbreitete Infektion zurückgeht, oder etwa auf die Tatsache, dass Eltern zum Homeschooling zuhause bleiben und sich deswegen nicht am Arbeitsplatz oder unterwegs anstecken.

\section{Kinder und COVID-19}

Als Arzt ist man gewohnt, die Wahrscheinlichkeiten von Nutzen und Risiken mit deren Effektstärken zu multiplizieren, um alternative Möglichkeiten therapeutischer Strategien rational abzuwägen. Alternativlos ist für uns alle nur der Tod, die Wege dorthin für jeden Einzelnen dagegen fast nie. Mit Ausnahme einer frühen chinesischen Studie [7], deuten die in einer Reihe von Untersuchungen aus verschiedenen Ländern gesammelten Erkenntnisse darauf hin, dass die Wahrscheinlichkeit, dass Kinder während der aktuellen Pandemie an Corona sterben, verschwindend gering ist [3, $28,31,37,50,55,62,82,105,109]$. Das SARS-CoV-2 Virus stellt 


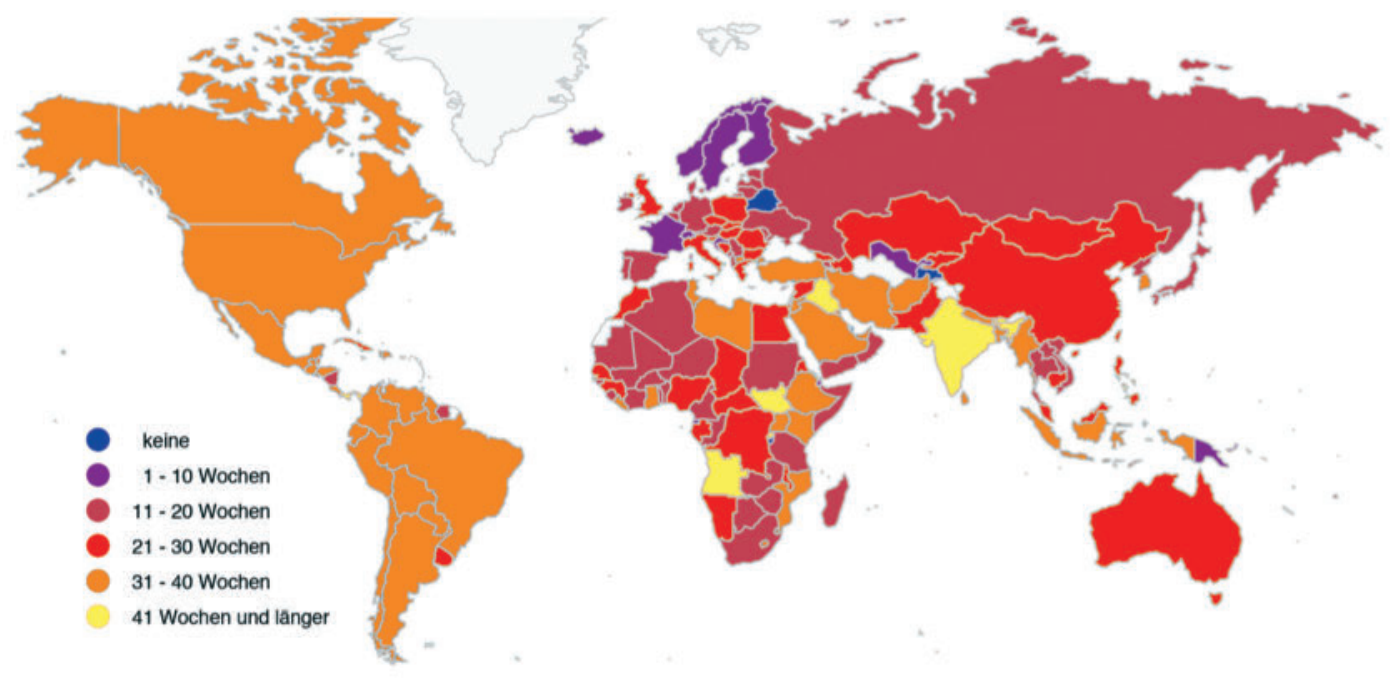

Abb. 1 Ort und Dauer der Schulschließungen weltweit (nach der UNESCO-Pressemitteilung vom 25. Januar 2021; https://en.unesco.org/ news/unesco-figures-show-two-thirds-academic-year-lost-average-worldwide-due-covid-19-school; Zugriff am 16. Februar 2021; modifizierte Einfärbung zur Darstellung einer „Heatmap“, wobei wärmere und hellere Farben längere Schulschließungen anzeigen). Die Schulen waren im Durchschnitt 3,5 Monate (14 Wochen) seit Ausbruch der Pandemie vollständig geschlossen. Lokale Schulschließungen dauerten im Durchschnitt 5,5 Monate (22 Wochen), was zwei Dritteln des akademischen Jahres entspricht.

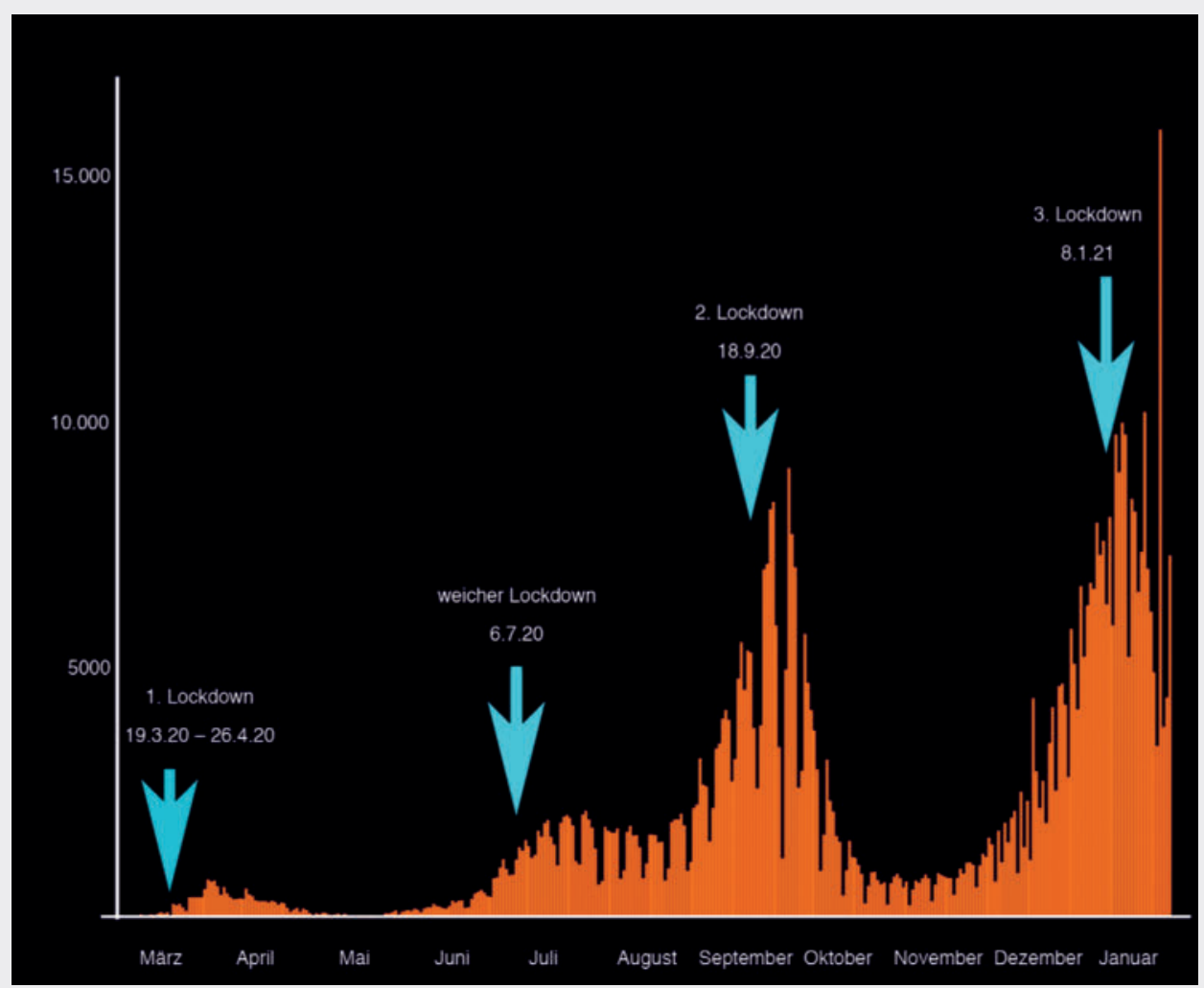

- Abb.2 Anzahl der täglichen Neuinfektionen in Israel von März 2020 bis Januar 2021 (modifizierter Screenshot aus dem Dashboard der Johns Hopkins University, aufgenommen am 29. Januar 2021). Der Zeitpunkt von 3 sehr strengen Abriegelungen („harte“ Lockdowns) und einigen Einschränkungen bei sozialen Aktivitäten im Sommer („weicher“ Lockdown) wurde vom Autor eingefügt, um die Wirksamkeit dieser Maßnahmen zu demonstrieren. Die Auswirkungen der Impfungen sind nicht zu erkennen. 
- Tab. 1 Wirksamkeit verschiedener Maßnahmen der Regierungen zur Eindämmung des Infektionsgeschehens während der Corona-Pandemie (nach Daten aus [8]).

\begin{tabular}{|l|l|}
\hline Maßnahme & $\begin{array}{l}\text { Effekt (Reduktion } \\
\text { des R-Wertes in } \\
\text { Prozent) }\end{array}$ \\
\hline Versammlungen mit höchstens 1000 Personen & 22 \\
\hline Versammlungen mit höchstens 100 Personen & 34 \\
\hline Versammlungen mit höchstens 10 Personen & 41 \\
\hline Einige Geschäfte geschlossen & 18 \\
\hline $\begin{array}{l}\text { Nahezu alle nicht lebenswichtigen Geschäfte } \\
\text { geschlossen }\end{array}$ & 26 \\
\hline \begin{tabular}{l} 
Schulen und Universitäten geschlossen \\
\hline Zusätzlicher Effekt von Ausgangsbeschränkun- \\
gen
\end{tabular} & 38 \\
\hline
\end{tabular}

damit für Kinder praktisch keine Gefahr dar. Ein von den US-amerikanischen Centers for Disease Control and Prevention (CDC) im Oktober 2020 veröffentlichter Bericht [28] beschreibt die demografischen Merkmale von 114411 COVID-19-assoziierten Todesfällen im Zeitraum vom 1. Mai bis 31. August. Das relative Risiko, an COVID-19 zu sterben, liegt in den 3 jüngsten Altersgruppen (Säuglinge, Kleinkinder/Vorschulkinder und Schulkinder/Jugendliche) unter $0,1 \%$ ( Tab. 2).

Mehrere Studien lieferten weitere Belege dafür, dass Kinder die Infektionsdynamik kaum beeinflussen. Eine am 22. Januar 2021 veröffentlichte Studie aus Südwestdeutschland untersuchte im April und Mai 2020 bei 2482 Kindern im medianen Alter von 6 Jahren (Bereich: 1-10) und einem entsprechenden Elternteil ( $n=2482$ ) die Prävalenz der Infektion mittels Reverse-Transkriptase-Polymerase-Kettenreaktion aus Abstrichen des Nasen-Rachenraums sowie SARS-CoV-2-lgG-Antikörper im Serum mittels Enzyme-Linked-Immunosorbent-Assay und Immunfluoreszenz. Sie fanden eine niedrige Seroprävalenz, mit signifikanten Unterschieden zwischen Eltern (1,8\%) und Kindern (0,6\%). Unter den 56 Familien mit mindestens einem seropositiven Kind oder Elternteil waren seropositive Eltern, die mit seronegativen Kindern zusammenlebten, 4,3-fach $(95 \% \mathrm{Cl}, 1,19-15,52)$ häufiger als seropositive Kinder, die mit seronegativen Erwachsenen zusammenlebten, was darauf hindeutet, dass Infektionen von Kindern auf Eltern viel unwahrscheinlicher sind als von Eltern auf Kinder [109]. Eine am 26.1. 2021 publizierte Studie aus Bayern, die im Sommer/Herbst 2020 über einen Zeitraum von 12 Wochen 3169 Abstriche von Kindern in Kitas und Grundschulen auf SARS-CoV-2 untersuchte, zeigte in nur 2 Fällen ein positives Ergebnis im rRTPCR-Test, obwohl im gleichen Zeitraum die Fallzahlen in der Gesamtbevölkerung stark angestiegen waren [37]. Nach den Angaben des Robert Koch-Instituts (RKI) befanden sich in Deutschland unter den gut 50000 an oder mit COVID-19-Verstorbenen, 9 Kinder und Jugendliche [30]. Die besonders hochrangig publizierten Daten aus Schweden, die zunächst deutlich für das Offenbleiben der Schulen zu sprechen schienen, bleiben in ihrer Aussagekraft
- Tab.2 Das Alter von 114411 Personen, die im Zeitraum vom 1. Mai bis 31 . August 2020 in den USA an COVID-19 verstorben sind (nach Daten aus [28])

\begin{tabular}{|l|l|}
\hline Alter (Jahre) & Todesfälle (\%) \\
\hline$<1$ & $<0,1$ \\
\hline $1-4$ & $<0,1$ \\
\hline $5-17$ & $<0,1$ \\
\hline $18-29$ & 0,5 \\
\hline $30-39$ & 1,4 \\
\hline $40-49$ & 3,5 \\
\hline $50-64$ & 16,4 \\
\hline $65-74$ & 21,7 \\
\hline $75-84$ & 26,0 \\
\hline$\geq 85$ & 30,4 \\
\hline unbekannt & $<0,1$ \\
\hline
\end{tabular}

unklar. ${ }^{1}$ Insgesamt zeigen die schwedischen Daten jedoch, wie die genannte US-amerikanische Studie [62], eine sehr geringe Mortalität bei Kindern. Sie zeigen auch, dass die Gefährdung des Lehrpersonals gering ist, obwohl in schwedischen Schulen das Tragen von Gesichtsmasken nicht verpflichtend angeordnet war. In einem Editorial mit dem Shakespeareschen Titel „To Spread or Not to Spread SARS-CoV-2 - Is That the Question?" stellt Sean O'Leary im Fachblatt JAMA Pediatrics am 22. Januar 2021 die inzwischen gut etablierte Tatsache klar, dass die Hospitalisierungs- und Sterberaten bei Kindern weitaus geringer sind als bei Erwachsenen. Außerdem machen Kinder einen geringeren Anteil der Fälle aus, als man aufgrund der Größe ihres Anteils an der Bevölkerung erwarten würde [3, 109].

Da Kinder nach den vorliegenden Erkenntnissen nur selten erkrankt sind, geht von ihnen auch ein geringeres Risiko aus als von erwachsenen Menschen ( $\triangleright$ Abb. 3). Hinzu kommt, dass Kinder statistisch eher von Erwachsenen angesteckt werden, als dass sie Erwachsene anstecken. Damit geht von Kindern prinzipiell ein geringeres Risiko aus als von Schülern der Klassen 8 bis 13 (Mittelstufe und Oberstufe) oder von Erwachsenen.

Wenn Kinder in nur geringem Maß an der Ausbreitung von COVID-19 beteiligt sind [75], warum werden Schulschließungen dann von politischen Entscheidungsträgern so gerne gegen die Ausbreitung der COVID-19-Pandemie eingesetzt? Wie O'Leary aufzeigte, liegt dies möglicherweise an deren Schwarz-Weiß-Denken. Aus seiner Sicht wird die Frage „Können Kinder COVID-19 verbreiten“ oft ohne Zögern mit „Ja“ beantwortet, obwohl die Chancen für ein solches „Ja“ faktisch sehr gering sind. Wie oben erwähnt, sind

1 Es ist bekannt, dass Schweden im Hinblick auf die Corona-Pandemie einen Sonderweg ging. Die Grundschulen (bis 9. Klasse) blieben im ganzen Jahr 2020 offen. Eine am 6.1.2021 im New England Journal of Medicine publizierte Studie stellte fest, dass nur wenig Hinweise auf eine Beteiligung von Kindern am Infektionsgeschehen vorlägen [62]. Mittlerweile wurde diese Studie jedoch kritisiert, die Daten seien gezielt ausgewählt und dargestellt worden, um den schwedischen Sonderweg zu rechtfertigen [116]. So gab es in Schweden durchaus COVID-19-Ausbrüche an Schulen $[5,115]$. 

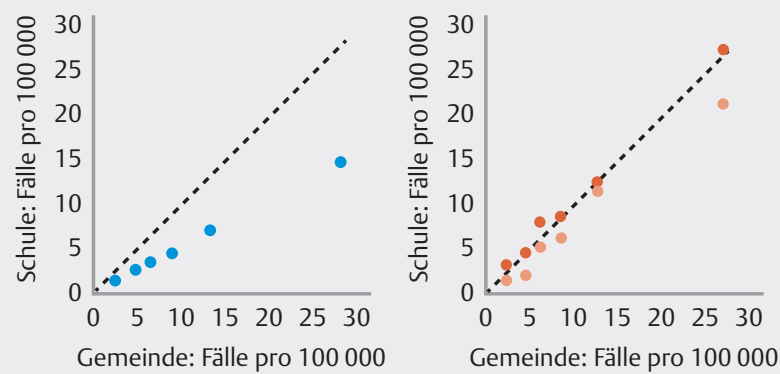

- Abb. 3 Rate der Infektionen mit COVID-19 in Gemeinden (Y-Achse) und den dortigen Schulen (X-Achse) im Staat New York (nach Daten aus [75]). Links: Kinder in den Klassen 1 bis 7 (Grundschule und Unterstufe; blaue Punkte) sind nur etwa halb so häufig infiziert wie die Mitglieder ihrer Gemeinde. Rechts: Jugendliche Schüler (orange Punkte) bzw. erwachsene Lehrer (rote Punkte) sind ebenso infektiös wie die Mitglieder der Gemeinde.

in der medizinischen Praxis Ja-Nein-Entscheidungen zwar binär, die Optionen müssen jedoch immer gewichtet werden. Daher ist „Ja, Kinder können COVID-19 verbreiten“ nicht gleichbedeutend mit „Wir sollten die Schulen schließen.“ Zum Vergleich: „Ja, bei der OP eines akuten Blinddarms kann man sterben" wird in der Medizin keineswegs gleichgesetzt mit „Wir operieren nicht“. Treiben wir den Gedanken etwas weiter: Im Jahr 2019 verunglückten 27742 Kinder (unter 15 Jahren) im Straßenverkehr, von denen 55 verstorben sind - etwa ein Drittel davon auf dem Weg zur Schule oder von dort nach Hause. Der Schulweg ist damit für Kinder deutlich gefährlicher als COVID-19. Warum schließen wir dann deswegen nicht die Schulen?

\section{Kinder im Lockdown: Nachgewiesene} Schäden

Gegenüber der äußerst geringen Wahrscheinlichkeit, mit der Kinder an COVID-19 erkranken oder gar versterben, und der eher geringen Bedeutung von Schulschließungen auf die Infektionsdynamik ist, die Wahrscheinlichkeit der Entwicklung von Übergewicht aufgrund von weniger körperlicher Aktivität und mehr Bildschirmmedienkonsum in Zeiten geschlossener Schulen groß. Nach einer Studie der Deutschen Angestelltenkrankenkasse (DAK) verbrachten Kinder und Jugendliche während des ersten Lockdown täglich 7 Stunden anstatt 5 Stunden mit Computerspielen und Social Media. Dabei wurden Fernsehen, die Nutzung von Videos oder Streamingdiensten wie Netflix gar nicht erfasst, d. h. diese Zeiten kommen noch hinzu [18, 101].

\section{Die Inaktivitätspandemie}

Man spricht seit etwa einem Jahrzehnt von einer weltweiten Inaktivitätspandemie [52]. Die damit einhergehenden Herz-Kreislauf-Erkrankungen sind in der westlichen Welt die häufigste Todesursache. Die Folgekosten weltweiter Inaktivität wurden schon vor einigen Jahren mit über 50 Milliarden US\$ jährlich („vorsichtig“, wie die Autoren hervorheben) geschätzt [20]. Es folgt auch: Die Wahrscheinlichkeit, an den Folgen des Lockdowns zu versterben, ist für Kinder und Jugendliche deutlich höher als das entsprechende Risiko von COVID-19, wie Studien aus Ländern wie Kanada, der Türkei, Portugal, China und Deutschland berichten [18, 23, 68, 85, 106, 132]. Was diese Erkenntnisse konkret bedeuten, sei durch einen Auszug aus einer E-Mail illustriert, die mir eine Lehrerin an einem Berliner Gymnasium, selbst Mutter zweier kleiner Kinder (2. und 6. Klasse), am 15. Mai 2020 schrieb, also nach der Zeit der Schulschließungen: „Die Schüler, die ich jetzt für einige wenige Stunden wiedersehen durfte, haben die 7 Wochen fast ausnahmslos spielend vor dem Rechner verbracht. Sie waren im Schnitt 5-10 Kilo schwerer [... und] sie sprechen davon, dass ihnen ihr Sport fehlt, sie aber aus Angst das Haus nicht verlassen haben. Die meisten meiner Schüler sind 17-19 Jahre alt. Ich finde es in diesem Alter besonders bedenklich, die Schüler in digitale Welten abdriften zu lassen. Hier wird auch der Grundstein für Suchtbiografien gelegt, die später schwer zu korrigieren sein werden“ [99].

Die negativen gesundheitlichen Auswirkungen von Übergewicht (BMI> 25) oder gar Adipositas (BMI>30) im Kindes- und Jugendalter sind hinlänglich bekannt [89, 91, 104, 107, 111]. Hinzu kommt die leider nur zu bekannte Tatsache, dass Gewicht viel leichter zu- als abgenommen wird und daher aus übergewichtigen Kindern und Jugendlichen sehr oft übergewichtige Erwachsene werden. Die Folgen - Bluthochdruck und Diabetes (Typ II) - und dass damit langfristig erhöhte Risiko für Schlaganfall, Herzinfarkt und Krebserkrankungen sind gravierend [77]. Eine 2012 in der medizinischen Fachzeitschrift Lancet veröffentlichte Arbeit bezifferte die globalen gesundheitlichen Folgen von Inaktivität auf jährlich 5 Millionen Todesfälle [122]. Darüber hinaus wurde berechnet, dass eine Reduzierung der körperlichen Inaktivität um 10\% weltweit 533000 Todesfälle pro Jahr abwenden könnte; eine Reduktion um $25 \%$ würde mehr als 1,3 Millionen Todesfälle abwenden [57]. Angesichts dieser Zahlen lässt sich der Effekt einer deutlich verringerten körperlichen Aktivität bei 1,6 Milliarden Kindern im Umfang von mittlerweile 2 Lockdowns (d. h. mehr als 3 Monaten in den meisten Ländern), nur erahnen, aber eines ist schon jetzt klar: Er wird nicht gering sein.

\section{Die Myopie-Pandemie}

Myopie oder Kurzsichtigkeit ist eine optische Fehlsichtigkeit des Auges, bei der das Licht vor und nicht auf der Netzhaut fokussiert ist. Die Brechkraft der Linse ist für die Länge des Auges also zu groß. Kurzsichtigkeit wird in Dioptrien gemessen, und weil sie durch eine Zerstreuungslinse korrigiert wird, mit einem Minuszeichen versehen. Kleinere Werte als -0,5 Dioptrien ( $\mathrm{dpt}$ ) bezeichnet man als Myopie. Bei noch kleineren Werten (also unter $-5 \mathrm{dpt}$ ) spricht man von hoher Myopie. Die meisten Fälle resultieren aus einer Fehlentwicklung des Augapfels, die sich einstellt, wenn man während der ersten beiden Lebensjahrzehnte zu viel Zeit mit dem Blick in die Nähe zubringt [ 92 , 121]. Das Auge hat einen eingebauten Mechanismus, der es so lange wachsen lässt, wie das durchschnittliche Bild auf der Netzhaut noch unscharf ist (weil das Auge noch zu kurz ist). Das Auge wächst also genau auf die Länge, die das Bild auf der Netzhaut in einen scharfen Fokus bringt $[93,118]$. Schaut der Mensch jedoch viele Stunden am Tag in die Nähe, wächst das Auge länger, da Licht, das von einem Punkt in kurzer Entfernung ausgeht (wie beim Lesen), weiter hinten im Auge fokussiert wird [97]. Vor mehr als 140 Jahren wurde entdeckt, dass Schulbildung (was viel Lesen impliziert) Kurzsichtigkeit 
verursacht [117]. Mittlerweile zeigen viele groß angelegte Studien, dass die in der Schule verbrachte Zeit mit einer Zunahme der Kurzsichtigkeit einhergeht $[67,71,72,81]$. Im Gegensatz dazu ist die Zeit im Freien - der Blick in die Ferne bei hellem Licht - bei Kindern und Studenten mit einer Abnahme der Myopie verbunden [36, 130].

$40 \%$ der Nordamerikaner sind von Myopie betroffen. Die Zahl der Fälle hat sich zwischen 1972 und 2004 verdoppelt und nimmt weiter zu [114]. In Europa lässt sich ähnlich wie in den USA die Myopie bei 42,2\% der Erwachsenen im Alter von 25 bis 29 Jahren feststellen und damit in dieser Altersgruppe fast doppelt so häufig wie bei Erwachsenen im Alter von 55 bis 59 Jahren [123, 124], die Zahl steigt [43]. AuBerhalb ophthalmologischer Kreise wissen jedoch nur wenige Menschen in westlichen Ländern von der Myopie-Pandemie [108]. Dies ist in Asien anders, wo die hohe und weiter steigende Prävalenz der Myopie vielen Menschen große Sorgen bereitet. In einer 15-jährigen bevölkerungsbasierten Längsschnittstudie an 43858 Oberschülern aus einer Stadt im Osten Chinas stieg die Prävalenz der Myopie von 79,5\% im Jahr 2001 auf 87,7\% im Jahr 2015. Die Prävalenz der hohen Myopie stieg im gleichen Zeitraum von 7,9\% auf 16,6\% [12]. Eine weitere Studie an 6364 17-Jährigen bezifferte den Anteil der Myopie auf $80 \%$ und den der hohen Myopie auf 14\% [128]. In Südkorea ergab eine Querschnittsstudie bei 23616 19-jährigen Männern eine Prävalenz von 96,5\% für Myopie und 21,6\% für hohe Myopie [48]. Myopie stellt einen Risikofaktor für alle 4 Hauptursachen von Erblindung im höheren Alter dar [16]:

- Makuladegeration (atrophische myope Makulopathie/Retinopathie), eine noch immer unbehandelbare, langsam fortschreitende Sehbehinderung, bei der sich der Sehverlust aufgrund einer Atrophie des retinalen Pigmentepithels entwickelt. Sie gehört zu den häufigsten Ursachen für Erblindung. Abhängig vom Grad der Myopie ist das Risiko der Makuladegeration im Durchschnitt um das 18-Fache erhöht (OR 2,2 bei $-1 \mathrm{dpt}$ bis $-3 \mathrm{dpt}$; OR 349 bei< $-9 \mathrm{dpt}$ ).

- Ein ähnlicher Zusammenhang wurde zwischen zunehmender Myopie und Netzhautablösung beobachtet, wobei das Risiko im Durchschnitt bei jeder Myopie um das 7,8-Fache erhöht ist. (OR 4,4 bei -1 bis $-3 \mathrm{dpt}$; OR 9,9 bei -3 bis $-8 \mathrm{dpt}$ ).

- Für ein Glaukom („grüner Star“), d. h. einen pathologisch erhöhten Augeninnendruck, wurde eine 2,3-fache Erhöhung bei niedriger Myopie (bis -3 dpt) und eine 3,3-fache Erhöhung bei mittlerer und hoher Myopie (unter $-3 \mathrm{dpt}$ ) festgestellt.

- Für Katarakte („grauer Star“) verschiedener Art berichten mehrere Studien mit unterschiedlichen Verfahren erhöhte ORs im Bereich von 1,4 bis 2,1 für geringe Myopie (-0,5 dpt bis $-2 \mathrm{dpt}$ ), 3,1 für moderate Myopie (-2 dpt bis -4 dpt), 5,5 für hohe Myopie (-4 dpt bis -6 dpt) und 12,3 für Myopie über -6 dpt [25].

Zusammengenommen zeigen diese Daten deutlich, warum Myopie weithin als eine „unterschätzte globale Herausforderung für das Sehen" [38] angesehen wird.

In Deutschland lesen nur 34\% der Schulkinder regelmäßig Bücher (im Durchschnitt etwa eine Stunde pro Tag; [47]), d. h. das Lesen von Büchern dürfte für die Entwicklung der Kurzsichtigkeit bei den meisten Kindern und Jugendlichen nur eine geringe Rolle spielen. Da Smartphones jedoch nicht nur die am weitesten verbreiteten und am häufigsten genutzten digitalen Bildschirmmedien sind [79], sondern auch die kleinsten Bildschirme haben (die einen sehr nahen Blick bis auf ca. $25 \mathrm{~cm}$ erfordern, im Vergleich zum Lesen von Büchern mit einem Abstand von etwa $50 \mathrm{~cm}$ ), ist der Blick auf Smartphones für 5 Stunden pro Tag oder mehr bei vielen jungen Menschen ein Problem [61]. In der zweiten Hälfte des letzten Jahrhunderts wurde in vielen entwickelten Ländern ein Anstieg des durchschnittlichen Bildungsniveaus beobachtet. Dieser Faktor allein kann jedoch nicht den Anstieg der Myopie erklären [123], da es in dieser Zeit auch eine zunehmende Nutzung von Bildschirmen (TV, Videos, Computer) und vor allem auch eine deutliche Abnahme der im Freien verbrachten Zeit gab. Eine Reihe von Autoren führt dies als Ursache für den Anstieg der Myopie an [33, 34, 36, 69, 70]. In jüngster Zeit wurden jedoch insbesondere Computernutzung und Smartphone-Nutzung als Risikofaktoren für Myopie identifiziert [1, 22, 24, 120].

Angesichts dieser Daten zur Myopie ist es nicht verwunderlich, dass in China eine bis zu 3-fache Zunahme der Wachstumsgeschwindigkeit der Augenlänge während der 4-monatigen Schulschließung und des Hausarrests während der COVID-19-Pandemie beobachtet wurden war [120]. In einer prospektiven Querschnittsstudie über 6 aufeinanderfolgende Jahre (2015-2020) wurde die Prävalenz der Myopie bei 123535 Kindern (6 bis 13 Jahre; 52,1\% Jungen) aus 10 Grundschulen untersucht. Es wurden Vergleiche zwischen dem Jahr 2020 (nach dem Lockdown) und den vorangegangenen 5 Jahren für jede Altersgruppe durchgeführt. Bei den schulischen Messungen der 6-, 7- und 8-jährigen Kinder im Jahr 2020 wurde eine erheblich stärkere Entwicklung in Richtung Myopie (ca. -0,3 dpt) festgestellt. Bei diesen Kindern war die Prävalenz der Myopie in den Messungen von 2020 höher als die höchste Prävalenz der Myopie in den Jahren bis 2015-2019 für Kinder im Alter von 6 (21,5\% vs. 5,7\%), 7 (26,2\% vs. $16,2 \%$ ) und 8 (37,2\% vs. 27,7\%) Jahren. Die Unterschiede bei Kindern im Alter von 9 bis 13 Jahren waren kleiner und statistisch nicht mehr signifikant. „Der Brechungsfehler jüngerer Kinder könnte empfindlicher auf Umweltveränderungen reagieren als der älterer Kinder, da sich die jüngeren Kinder in einer besonders vulnerablen Periode für die Entwicklung von Myopie befinden“, schließen die Autoren. Ein weiterer Grund, warum bei Kindern zwischen 9 und 13 Jahren nur eine geringere Zunahme zu beobachten war, könnte ein Deckeneffekt sein: Ihre Bildschirmzeit könnte bereits so hoch gewesen sein, dass der Lockdown nicht viel mehr Zeit und damit Myopie hinzufügen konnte [51].

Wie sollten diese Effekte bewertet werden? - Eine systematische Übersichtsarbeit und Metaanalyse von 145 Studien (veröffentlicht seit 1995) an insgesamt 2,1 Millionen Menschen schätzte die aktuelle Zahl der Menschen mit Myopie weltweit auf 1,4 Milliarden (23\% der Weltbevölkerung) und die Zahl der Menschen mit hoher Myopie auf 163 Millionen (2,7\%). Für das Jahr 2050 wurden Prognosen mit 4,8 Milliarden Menschen (etwa 50\% der Weltbevölkerung) mit Myopie und 938 Millionen Menschen (fast 10\% der Weltbevölkerung) mit hoher Myopie genannt [39]. Die Zunahme auf fast eine Milliarde Menschen mit hoher Myopie und das Ausmaß der resultierenden Blindheit werden in Asien sehr ernst genommen. China beispielsweise steht in Anbetracht der Tatsache, dass einer von 3 Patienten mit hoher Myopie meist schon vor dem Rentenalter erblinden wird, vor einem ernsten Problem der öffentlichen Gesundheit. Dies ist wahrscheinlich der Grund dafür, dass China am 15. Januar 2021 ein Smartphone-Verbot an allen Schulen in Kraft gesetzt hat ( $>$ Abb. 4). In einem Land mit 1,3 Milliarden Menschen 


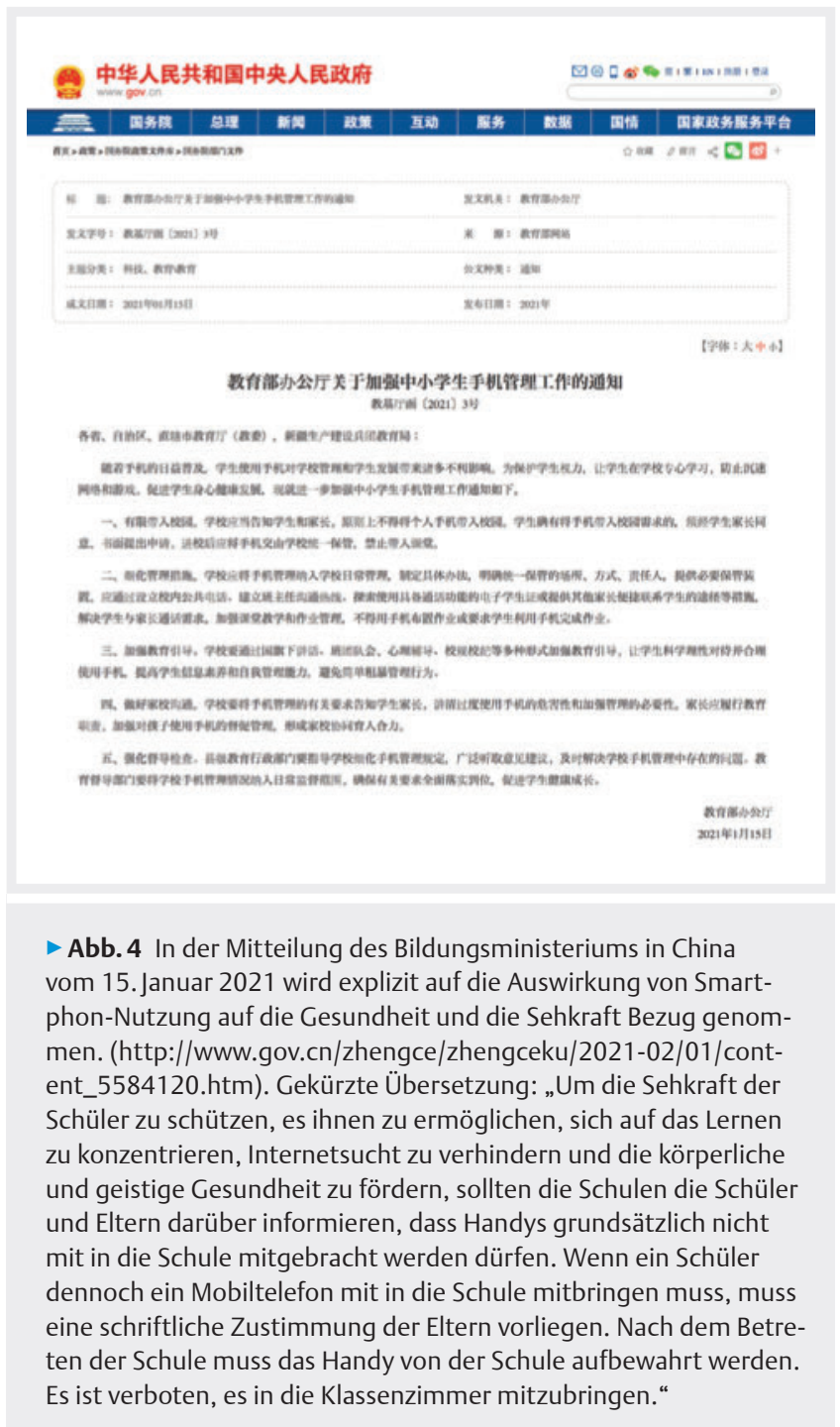

und einer 80 \%igen Prävalenz von Myopie in der jüngeren Bevölkerung, mit steigender Tendenz, scheinen die Kosten der Myopie in Form von menschlichem Leid und wirtschaftlicher Belastung solch drastische Maßnahmen zu rechtfertigen. ${ }^{2}$ Ein Großteil der übrigen Welt wird wahrscheinlich folgen.

\section{Störungen der seelischen Gesundheit: Gehirnentwicklung, Stress, gelernte Hilflosigkeit, Depression, Angst, Einsamkeit, Sucht und antisoziales Verhalten}

Eine zunehmende Anzahl von Studien berichtet über die psychologischen Auswirkungen der COVID-19-Krise auf die Allgemeinbevölkerung und insbesondere auch auf jüngere Menschen: Er-

2 Man muss hierzu wissen, dass in China das Smartphone beim Schulunterricht eine wesentliche Rolle spielte: Hausaufgaben wurden mit ihm nicht nur aufgegeben, sondern auch erledigt, Materialien wurden ausgegeben und der Unterricht an ihm und mit ihm durchgeführt. Das alles ist nicht mehr möglich, was zu erheblichen Problemen geführt hat. höhte Stresswerte, Depressionen (einschließlich Suizidrisiko; [64]), Angstzustände, Müdigkeit und verminderter Schlaf [95, 96, 110]. Bei Kindern und Jugendlichen sind diese negativen Auswirkungen gravierender, da die Gehirnentwicklung - ebenso wie die Augenentwicklung - in den ersten 2-3 Lebensjahrzehnten erfolgt und ihre Beeinträchtigung umso bedeutsamer ist, je jünger die Betroffenen sind. Die Entwicklung des Gehirns hängt von Erfahrungen ab: Zur Sprachentwicklung beispielsweise gehören das Zuhören, das Artikulieren und das gemeinsame Handeln im Dialog. Sie findet nicht durch passive Betrachtung von Bildschirmen statt. Auch die Beherrschung der Sprachlaute, die schon vor dem ersten Lebensjahr erfolgt, erfordert Lernen in sozialen Kontexten und nicht über Lautsprecher [53, 54]. Mitgefühl, Persönlichkeit, das Führen von Dialogen und der Austausch von Argumenten, das Lösen von Konflikten und vieles mehr hängt von der Zeit ab, die in der Gemeinschaft mit Familie und Freunden verbracht wird. Empathie und Selbstvertrauen entstehen durch gelungene emotionale Interaktionen und durch Projekte und Erfahrungen von Selbstwirksamkeit im Alltag, wie Langzeitstudien zur menschlichen Entwicklung zeigen [88].

Anstatt Sprachentwicklung, Persönlichkeitsbildung und sozialem Lernen erfolgt im Lockdown vor allem das Erlernen von Hilflosigkeit, denn man lernt, dass man der Pandemie ausgeliefert ist, an ihrem Verlauf nichts ändern kann und ständig Angst vor der eigenen Ansteckung bzw. der Ansteckung anderer hat. Auch die verordnete soziale Isolation führt nachweislich zu Stress, der sich wiederum negativ auf die Gesundheit auswirkt - je länger er anhält, umso mehr. „Gelernte Hilflosigkeit“ ist das bekannteste psychologische Modell für Stress und Depression. Da jüngere Menschen schneller lernen als Ältere, erlernen sie auch schneller Hilflosigkeit. Hinzu kommt, dass jüngere Menschen weniger Lebenserfahrungen haben, die ihren Eltern und Großeltern helfen, Zeiten der sozialen Isolation, des Stresses und der wirtschaftlichen Not zu überstehen. Unter normalen Umständen liegt die weltweite Prävalenz von psychischen Störungen bei Kindern und Jugendlichen bei 13,4\%, wie eine Metaanalyse zeigt [82]. Mehrere Studien ergaben mittlerweile, dass psychische Probleme in der Pandemie zugenommen haben, nicht so sehr wegen des neuen Coronavirus, sondern wegen der Maßnahmen des Lockdowns, die zur Eindämmung der Infektionen ergriffen wurden. Die erste dieser Studien kam aus China und berichtete über eine Zunahme von Stress, Angst und Depression [46]. Xie und Mitarbeiter [129] fanden heraus, dass während der Pandemie in China 23 \% der Kinder der 2. bis 6. Klasse depressive Symptome hatten und 19\% unter Angstzuständen litten. In einer Querschnittsstudie mit 8079 chinesischen Gymnasiasten (im Alter von 12-18 Jahren) während der COVID-19-Epidemie lag die Prävalenz von depressiven Symptomen, Angstsymptomen und einer Kombination aus depressiven und Angstsymptomen bei 43,7\%, 37,4\% bzw. 31,3\% der chinesischen Schüler [132].

Eine repräsentative Online-Befragung wurde in Deutschland unter 1586 Familien mit 7- bis 17-jährigen Kindern und Jugendlichen zwischen dem 26. Mai und dem 10. Juni 2020, also wenige Wochen nach dem ersten Lockdown, durchgeführt. Die Autoren konnten ihre Ergebnisse mit Daten aus der bundesweiten, repräsentativen Längsschnitt-Kohortenstudie ( $n=1556)$ vergleichen, die vor der Pandemie durchgeführt worden war. Die Kinder und Jugendlichen hatten eine signifikant geringere gesundheitsbezogene Lebensqualität (40,2\% vs. 15,3\%), mehr psychische Proble- 
me (17,8\% vs. 9,9\%) und höhere Angstwerte (24,1\% vs. $14,9 \%)$ als vor der Pandemie [87]. Ein webbasiertes Interview bei 932 Universitätsstudenten zeigte ebenfalls während der Pandemie erhebliche psychische Probleme, wobei die emotionalen Schwierigkeiten bei Frauen und Studenten im Grundstudium deutlicher waren als bei Männern und Postgraduierten. Die psychische Belastung stand hauptsächlich im Zusammenhang mit den folgenden von den Teilnehmern wahrgenommen Stressoren: akademische Zukunft, Aufgabenüberlastung, Verschlimmerung von zwischenmenschlichen Konflikten und Einschränkungen bei angenehmen sozialen Kontakten; der Zusammenhang mit der Ausbreitung der Krankheit und ihren Folgen für die körperliche Gesundheit war vergleichsweise deutlich geringer [76].

TV-Konsum von Kindern und Jugendlichen hat bekanntermaßen schädliche Auswirkungen wie Übergewicht und geringe Fitness im Erwachsenenalter [32], Aufmerksamkeitsstörungen [58, 73] und geringere Empathie gegenüber Eltern und Gleichaltrigen [88] bis hin zu erhöhtem antisozialem Verhalten [90]. Während des Lockdowns hat der Fernsehkonsum zugenommen, weswegen man davon ausgehen kann, dass diese negativen Effekte verstärkt auftreten. Die körperlichen Auswirkungen wurden schon als „Covibesity“ bezeichnet [49], wozu ungesunde Ernährung während des Lockdowns (mehr Kartoffelchips, rotes Fleisch und zuckerhaltige Getränke; [80]), verringerte körperliche Aktivität und mehr Bildschirmzeit gemeinsam beitragen. All diese Effekte sind bei benachteiligten Kindern wie Kindern aus Familien mit niedrigem Einkommen oder Eltern mit niedrigem Bildungsniveau stärker ausgeprägt. Teil des Lockdowns war die Aufforderung, körperlichen Abstand zu anderen Menschen zu halten, um Infektionen zu vermeiden. Leider wurde dies als „soziale Distanzierung“ bezeichnet. Es wäre besser gewesen, wenn man von Anfang an von „körperlicher Distanzierung “ gesprochen hätte, um zu betonen, dass man auch bei räumlicher Trennung sozial verbunden bleiben kann. Noch besser wäre der vom sozialen Neurowissenschaftler Jamil Zaki aus Stanford vorgeschlagene Ausdruck des „distant socializing“ gewesen [19], um sicherzustellen, dass es keineswegs um die Forderung nach mehr Einsamkeit geht. - Aber so wurde das Ganze leider von vielen Menschen verstanden. Einsamkeit und soziale Isolation betrafen schon vor der Pandemie eine große Anzahl von Menschen. jüngsten Schätzungen zufolge nahm die Einsamkeit während des Lockdowns nochmals um $20-30 \%$ zu [41].

Auch wenn die meisten Menschen der Meinung sind, dass ältere Menschen am meisten von Einsamkeit betroffen, sind es tatsächlich die jüngeren Menschen, die am meisten leiden [11]. Soziale Isolation und Einsamkeit verursachen sowohl kurzfristige (Suizid und häusliche Gewalt) als auch langfristige Auswirkungen in Bezug auf eine Vielzahl von chronischen Krankheiten [40]. Das Risiko für diese Krankheiten steigt, womit ein erheblicher Anstieg des Sterberisikos einher geht. Auch das Risiko, an COVID-19 zu erkranken, ist bei einsamen Menschen sehr wahrscheinlich erhöht [14], weil Einsamkeit zu Stress führt und daher die Immunabwehr beeinträchtigt. Was die Auswirkungen von Einsamkeit und sozialer Isolation bei Kindern und Jugendlichen betrifft, so zeigt eine Übersicht zu 83 Arbeiten [59], dass sie während und nach erzwungener Abgeschlossenheit und Isolation häufiger an Depressionen und Angstzuständen leiden. Die langfristigen Folgen hiervon werden erst durch zukünftige Forschung ans Licht kommen.
Zusammenfassend lässt sich Folgendes festhalten: Vom Baby bis zum Universitätsstudenten ist die physische und psychische Gesundheit durch Lockdown-Maßnahmen gefährdet. Babys leiden unter mehr Gewalt und Stress ihrer Eltern, Kleinkinder vermissen ihre Freunde, Vorschulkinder noch mehr. Kinder vermissen die Schule (ja, das tun sie wirklich!), ebenso wie Heranwachsende, die möglicherweise zum ersten Mal die Bedeutung von Lehrern für das Lernen erkennen. Hinzu kommt, dass Übergewicht und mangelnde Bildung sich gegenseitig verstärken [14]. Leider sind auch die Bedenken der zitierten Lehrerin hinsichtlich der Suchtentwicklung bei ihren Schülern nur allzu berechtigt. Studien zeigen, dass die Corona-Pandemie das Suchtproblem verschärft hat [133, 134].

\section{Bildungsverlust, Vergrößerung des SES-Gefälles, wirtschaftliche Not und Ungleichheit}

Kurzfristig führen Schulschließungen zu einem Rückgang der Lernleistung und zu einer Zunahme von Schulabbrüchen, Kinderarbeit, Gewalt gegen Kinder und Teenagerschwangerschaften [60]. Da viele Kinder ihr Frühstück oder Mittagessen in der Schule bekommen, führen Schulschließungen zudem in vielen Ländern zu Ernährungsunsicherheit und Unterernährung [13, 113]. Diese Folgen wurden bereits während der Ebola-Epidemie von 2014-2016 beobachtet [2]. Wirtschaftliche Abschwünge und Arbeitslosigkeit aufgrund des Lockdown werden mit einer verminderten psychischen Gesundheit von Erwachsenen in Verbindung gebracht, die sich auch auf die psychische Gesundheit von Kindern und Jugendlichen ungünstig auswirken kann [27]. Langfristig führen die genannten gravierenden Auswirkungen auf Gesundheit und Bildung zu anhaltenden sozioökonomischen und Mortalitätsunterschieden zwischen Arm und Reich [6, 83, 113].

Das Ausmaß des durch die Schulschließungen während der Corona-Pandemie verursachten Bildungsrückstands zeigt eine Studie an 350000 Schülern in Holland. Im digitalen Distanzunterricht wurde fast nichts gelernt, obwohl es in Holland seit Jahren keinen Mangel an Computern und Erfahrungen mit Computern im Unterricht gibt [21]. Genau aus diesem Grund haben die Autoren bewusst Daten aus Holland analysiert. Das Argument, man habe keine Erfahrung mit Computern und zu wenig Geräte, trifft dort nicht zu. Die Tatsache, dass man in Deutschland davon spricht, das Sitzenbleiben auszusetzen, geringere Anforderungen zu stellen, den Schülern und Studenten das Jahr nicht (auf die Schul-, Studien- oder Ausbildungszeit) anzurechnen oder es vielleicht einfach gar nicht zu bewerten und zu wiederholen, lässt erahnen, wie ernst die Situation ist. Da Kinder in der Schule sehr schnell sehr viel lernen, leidet ihre Bildung enorm, wenn ein halbes Schuljahr (oder mehr) ausfällt. Noch wichtiger ist, dass geschlossene Schulen die Kluft zwischen stärkeren und schwächeren Schülern deutlich vergrößern, denn einem guten Schüler kann man bei Schulausfall beispielsweise ein Buch in die Hand drücken, das dieser dann auch mit Gewinn lesen wird. Ein schwacher Schüler nicht. Je stärker ein Schüler ist, desto besser ist seine Aufmerksamkeit und Konzentrationsfähigkeit - auch und gerade beim Lernen am Computer. Langfristig führt die Schulschließung im Rahmen des Lockdowns also zu einer sozialen Krise durch verstärkte soziale Ungleichheit, der mit entsprechenden politischen Maßnahmen begegnet werden muss. 
Auch aus diesen Gründen ist Begrenzung von Schulschließungen auf das absolut notwendige Minimum zu fordern.

Ludger Wößmann, Professor für Bildungsökonomie an der Ludwig-Maximilians-Universität in München und seit 2004 Direktor des dortigen ifo-Zentrums für Bildungsökonomie, untersucht seit mehr als 2 Jahrzehnten den Zusammenhang zwischen Bildung und Wirtschaft. Dazu gibt es sehr belastbare Daten aus einer Vielzahl von Ländern über vergangene Schulabbrüche, Kurzschuljahre und neu eingeführte zusätzliche Schuljahre. „Nichts ist in der Bildungsökonomie so gut dokumentiert wie der Zusammenhang von Bildung und Einkommen “ [26]. Studien zeigen entsprechend: „Geht etwa ein Drittel eines Schuljahres an Lernen verloren, so geht dies über das gesamte Berufsleben gerechnet im Durchschnitt mit rund 3-4\% geringerem Erwerbseinkommen einher. Darüber hinaus zeigen Untersuchungen [...], dass ausbleibender Schulunterricht [...] den zukünftigen Arbeitsmarkterfolg dauerhaft schmälert" [26]. Erst kürzlich hat Wößmann die Auswirkungen von Schulschließungen in Deutschland bis zum Jahr 2100 (so lange leben jetzige Erstklässler etwa) hochgerechnet und auf 3300 Milliarden Euro beziffert [26]. Für den einzelnen Arbeitnehmer bedeutete die SchlieBung im letzten Frühjahr (12 Wochen) einen Verlust von $3 \%$ des Lebenseinkommens. Rechnet man den zweiten Lockdown hinzu, sind es 5,5\% des Lebenseinkommens. Bei den genannten negativen Auswirkungen von Schulschließungen auf die Gesundheit, die Bildung und den die gesamte Lebenszeit betreffenden wirtschaftlichen Erfolg junger Menschen handelt es sich um Fakten. Diese sind, ähnlich wie das Wetter, im Einzelfall schwer vorhersehbar, die Gesamtwirkung jedoch lässt sich ziemlich genau berechnen.

Ein Beispiel: Ob es am 12. November 2021 regnet, kann man heute nicht wissen; dass im Jahr 2021 in Deutschland etwa $75 \pm 10$ Liter Niederschlag pro Quadratmeter fallen werden, ist eine Tatsache. Wann in diesem Jahr in Deutschland wer an einem Schlaganfall oder Herzinfarkt verstirbt, weiß niemand; dass in diesem Jahr in Deutschland Schlaganfall und Herzinfarkt die häufigsten Todesursachen sein werden, ist eine Tatsache. Wie angeführt, handelt es sich bei den Auswirkungen von Schulschließungen auf die Bildung und den lebenslangen wirtschaftlichen Erfolg ebenfalls um gut belegte Fakten. Rechnet man die Auswirkungen von Corona mit denen der Schulschließungen anhand dessen, was wir bereits jetzt wissen, gegeneinander auf, dann ist unsere Behandlung der Krankheit verglichen mit der Krankheit das weitaus größere Übel.

\section{Testen und Impfen}

Im Gegensatz zum ersten Lockdown sind Tests und Mund-Nasen-Masken keine Mangelware mehr. Durch die möglichen einfachen und kostengünstigen Surveillance-Maßnahmen wie schnelle „Gurgeltests“, sollte es möglich sein, Infektionsherde - auch in Kitas und Schulen - frühzeitig zu erkennen und durch gezielte Quarantäne einzudämmen [66]. Da Abstriche von erfahrenem medizinischem Personal durchgeführt werden müssen, ist der Nachweis des Virus im Gurgelwasser auf der Ebene von Schulklassen möglich. Hierbei muss keineswegs für jeden Schüler ein einzelner Test verwendet werden. Wenn vielmehr die gesamte Klasse nach dem Gurgeln in den gleichen Eimer spuckt, wären solche klassenspezifischen Antigentests durchaus machbar und bezahlbar. Im US-amerikanischen Bundesstaat Massachusetts läuft hierzu gerade ein gro-
Bes Modellprojekt an mehr als 1000 Schulen [128]. Bei einer 7-Tage-Inzidenz von z. B. 80 Neuinfektionen pro 100000 Einwohner und einer Krankheitsdauer von 14 Tagen (wobei die Infektiosität tendenziell nicht die gesamten 14 Tage anhält) ist weniger als einer von 500 Einwohnern potenziell infektiös, d. h. 499 von 500 Bürgern sind nicht infektiös. Die Zahl der infizierten Kinder in Kindergärten und Grundschulen ist noch einmal deutlich geringer. Dem fast nicht vorhandenen potenziellen Nutzen für Kinder stehen reale Schließungen gegenüber, die Kindern nachweislich schaden. Aus meiner Sicht sind innovativere Strategien daher dringend erforderlich. Hierzu könnten Schnelltests beitragen, wenn man sich auf ein standardisiertes Vorgehen einigen könnte. Dies ist hierzulande bislang nicht erfolgt. In Berlin erhalten Schüler Tests für Zuhause, in Baden-Württemberg werden die Kinder während der ersten Stunde (warum nicht davor?) in kleinen Gruppen in einen anderen Raum zum Testen gebracht. Die Teilnahme ist freiwillig. Die Strategien beider Länder sind mithin löchrig bis ungenügend.

\section{Tests im Abwasser}

Gleich mehrere Studien zeigten bereits im Frühjahr 2020, dass das Virus im Abwasser nachgewiesen werden kann [35] und dass etwa ein Infizierter auf tausend Nichtinfizierte ausreicht, um diese Tests positiv werden zu lassen [56]. Dies wurde bereits genutzt, um Corona-Ausbrüche in Nachbarschaften [17] oder in Studentenwohnheimen [78] zu identifizieren. Selbst Genomanalysen zur Identifizierung neuer Mutanten sind im Abwasser möglich, wie Schweizer Wissenschaftler der ETH Zürich und des Instituts für Bioinformatik der Universität Lausanne kürzlich berichteten [45]. Da das Signal für SARS-CoV-2 im Abwasser auf lokaler Ebene 4 bis 20 Tage vor dem Signal neuer klinischer Fälle nachgewiesen werden konnte und Abwasser somit das Vorhandensein des Virus in einem bestimmten Gebiet früher anzeigt als klinische Fälle, stellt dies eine gute und sehr kostengünstige Möglichkeit zur Überwachung dar.

\section{Impfen}

In diesem Zusammenhang muss auch die Massenimpfung erwähnt werden. Während man sich auf die am stärksten gefährdeten Personen (Menschen über 80 Jahre) und diejenigen, die sie pflegen, konzentriert hat, wurde der Impfung von Erziehern und Lehrern keine hohe Priorität eingeräumt, obwohl sie dadurch sicher an ihren $\mathrm{Ar}$ beitsplatz würden zurückkehren können. „Es gibt nur eine Möglichkeit, die Schulen auch in einer dritten Welle zu einigermaßen sicheren Orten zu machen: indem man die Lehrer impft und gleichzeitig mindestens 2-mal in der Woche einen Schnelltest für alle Lehrer und Schüler durchführt, “ sagte der Präsident des Lehrerverbands Heinz-Peter Meidinger am 21.3.2021. Rechnen Sie bitte einmal nach: Jeder geimpfte Lehrer, der unterrichten darf, hat einen großen Einfluss auf die zukünftige Gesundheit, Bildung und den Wohlstand der vielen Kinder, die er oder sie unterrichtet. Wie beschrieben gibt es für Kinder nichts Besseres als den Präsenzunterricht. Mit dem Wohl der jungen Menschen wurde kaum argumentiert, wenn es um die richtige Impfstrategie ging [102]. Die Älteren hätten wir von vornherein besser schützen müssen: In Deutschland tauchte ein SARS-CoV-2-positiver Weihnachtsmann in einem Altenpflegeheim auf, steckte viele an und verursachte unnötige 
Todesfälle. Ebenso stellen Corona-Leugner und Verschwörungstheoretiker ein echtes Problem hinsichtlich der Ausbreitung von COVID-19 dar, weil sie sich unverantwortlich verhalten, indem sie sich nicht absetzen und keine Masken tragen. Außerdem gibt es immer noch Menschen, die auf unnötige Ausflüge und Partys nicht verzichten können. Aus diesem Grund und wegen einer hohen Prävalenz von COVID-19 zu Beginn des Jahres 2021 und der Möglichkeit weiterer tödlicher, mutierter Viren, bekämpfen wir diese Verantwortungslosigkeit nicht wirklich. Stattdessen versuchen wir, sie mit der noch größeren Verantwortungslosigkeit auszugleichen, die darin besteht, unsere von der Schule ausgesperrten Kinder als unsere Hauptverteidigungsstrategie einzusetzen.

\section{Diskussion: Die Zukunft der „Generation Corona“}

Kinder, Jugendliche und junge Erwachsene werden noch lange mit den Folgen der Corona-Pandemie auf ihre Lebensplanung und ihr Leben zu kämpfen haben. Nicht so sehr das Virus, sondern vielmehr unsere Maßnahmen zur Verhinderung von dessen Ausbreitung, werden langfristige negative Auswirkungen auf eine ganze Generation haben. Deren Gelegenheiten, das zu lernen, was junge Menschen lernen müssen, wurden reduziert, und die Zeiten, in denen sie lernten, was sie nicht lernen sollten - Hilflosigkeit, falsche Ernährung, viel zu viel Zeit vor Bildschirmen verbringen - waren deutlich erhöht. In dieser Übersichtsarbeit wurde gezeigt, dass die schädlichen Auswirkungen von Schulschließungen auf junge Menschen in Bezug auf Gesundheit, Bildung und Wohlbefinden viel nachteiliger sind, als gewöhnlich angenommen wird. Meiner Ansicht nach ist es daher wahrscheinlich, dass wir die jungen Menschen, die die Pandemie erlebt haben, bald als „Generation Corona“ bezeichnen werden: Einsam, unsicher, passiv und mit reduzierter Lebenserfahrung vor allem im sozialen Bereich. Die Pandemie und vor allem unsere Reaktion auf sie hat unweigerlich in den Köpfen und Körpern einer ganzen Generation für Jahrzehnte einen bleibenden Eindruck hinterlassen.

Um es nochmals deutlich zu sagen: Der erste Lockdown im Frühjahr 2020 war kaum vermeidbar, da wir nichts oder nur wenig über das Virus wussten, es nicht genügend Schutzausrüstung gab - nicht einmal für die Krankenhäuser und es zu wenig Testkapazitäten (PCR, Schnelltests) gab - von Impfungen gar nicht zu reden. Dies hat sich geändert: Wir wissen viel mehr, haben genügend Handschuhe, Desinfektionsmittel, Schutzleidung und Masken, testen und impfen. Daher können und sollten wir auf die Schließung von Kitas und Schulen verzichten. Was wir hierzu brauchen sind geimpfte Erzieher und Lehrer, kleine Klassen (bedeutet mehr Lernen und weniger Ansteckung), große, gut belüftete Räume, Abstandsregeln, Hygieneregeln, häufige, frühzeitige und konsequent strukturierte Tests (mit Quarantäne von identifizierten Fällen und ihren Kontaktpersonen) und das Tragen von Masken (insbesondere durch Lehrer) gemäß den WHO-Richtlinien sowie Maßnahmen zur Verhinderung der Übertragung von Personal auf Personal (Vermeidung von Menschenansammlungen in Lehrerzimmern; keine gemeinsamen Malzeiten von Erziehern bzw. Lehrern; [29]). Wir wissen jetzt viel mehr über die Übertragungsdynamik und die Maßnahmen zur Eindämmung der Corona-Pandemie als im März 2020 bei der Schließung der Schulen. Kinder, die 10 Jahre und jünger sind, haben sowohl eine geringere Wahrscheinlichkeit, sich mit SARS-CoV-2 zu infizieren, als auch eine geringere Wahrscheinlichkeit, es auf andere zu übertragen. Alle wirksamen Maßnahmen zur Eindämmung - Distanzierung, Masken, Tests, Impfen - können die Risiken weiter reduzieren und unsere Gesellschaft zurück zur Normalität bringen - einschließlich des Offenhaltens der Kitas und Schulen [42].

Ein letzter Gedanke scheint mir im Zusammenhang der Schulschließungen zur Bekämpfung der Pandemie von Bedeutung. Das Wohl künftiger Generationen wird ganz offensichtlich überhaupt nicht bedacht, obwohl es doch bei der „nächsten und noch viel größeren abzuwendenden Katastrophe“ - dem Klimawandel - eine wesentliche Rolle spielt. Diesen kann man überhaupt nur dann bekämpfen, wenn man das Wohl künftiger Generationen berücksichtigt, wie seit dem Brundtland Report aus dem Jahr 1987, also seit mehr als 30 Jahren, klar ist [103]. Wir müssen dieses Wohl quantifizieren, diskontieren (als den Wert der näheren Zukunft und der weiteren Zukunft gegenüber der Gegenwart mit einem Rabatt belegen, der auch negativ sein kann - für beides gibt es Argumente) und in alle gegenwärtigen und künftigen Planungen mit einbeziehen. Man kann sonst Klimawandel gar nicht sinnvoll in den Blick nehmen, geschweige denn Maßnahmen zur Eindämmung der Erderwärmung bewerten und abwägen. Das aber muss man tun, bevor man sie implementiert. Warum also das Wohl künftiger Generationen bei der einen Katastrophe (Klimawandel) fraglos und bei der anderen (Corona-Pandemie) überhaupt nicht betrachtet wird, entzieht sich aus meiner Sicht einer rationalen Erklärung. Wir müssen unsere Entscheidungen abwägen, um der verletzlichsten und gleichzeitig wertvollsten Ressource, die wir zur Bewältigung aller gegenwärtigen und zukünftigen Probleme haben, so wenig Schaden wie möglich zuzufügen: Unserer nächsten Generation. Aus meiner Sicht ist das Öffnen bzw. Offenhalten von Kitas und Schulen auch in schwierigsten Zeiten eine unserer wichtigsten Aufgaben.

\section{Literatur}

[1] Alvarez-Peregrina C, Sánchez-Tena MÁ, Martinez-Perez C, et al. The Relationship Between Screen and Outdoor Time With Rates of Myopia in Spanish Children. Front Public Health2020; 8: 560378

[2] Armitage R, Nellums LB. Considering inequalities in the school closure response to COVID-19. Lancet Glob Health 2020; 8: e644

[3] Bailey LC, Razzaghi H, Burrows EK, et al. Assessment of 135794 Pediatric Patients Tested for Severe Acute Respiratory Syndrome Coronavirus 2 Across the United States. JAMA Pediatr 2021; 175: 176-184

[4] BBC News. TV watching and online streaming surge during lockdown. August 5th, 2020 https://www.bbc.com/news/entertainment-arts-53637305; accessed February11th, 2021

[5] Besançon L, Steadson D, Flahault A. Open Schools, Covid-19, and Child and Teacher Morbidity in Sweden. N Engl J Med 2021; 10.1056

[6] Betz CL. COVID-19 and school return: The need and necessity. J Pediatr Nurs 2020; 54: A7-A9

[7] Bi Q, Wu Y, Mei S, et al. Epidemiology and transmission of COVID-19 in 391 cases and 1286 of their close contacts in Shenzhen, China: a retrospective cohort study. Lancet Infect Dis 2020; 20: 911-919

[8] Brauner JM, et al. Inferring the effectiveness of government interventions against COVID-19. Science 2021; 371(6531): 802

[9] Brückle M. Because of Corona, Netflix and Youtube decrease image quality in order to ease the load on communication networks [article in German] https://www.buffed.de/Netflix-Firma-259629/News/Streaming-Qualitaet-Corona-1345982/; accessed September 6th 2020 
[10] Brundtland Report. World Commission on Environment and Development der United Nations 1987 Our common future. www.are.admin. ch/are/de/home/nachhaltige-entwicklung/internationale-zusammenarbeit/agenda2030/uno-_-meilensteine-zur-nachhaltigen-entwicklung/1987--brundtland-bericht.html; abgerufen am 15.1.2021

[11] Bu F, Steptoe A, Fancourt D. Who is lonely in lockdown? Cross-cohort analyses of predictors of loneliness before and during the COVID-19 pandemic. Public Health 2020; 186: 31-34

[12] Chen M, et al. The increasing prevalence of myopia and high myopia among high school students in Fenghua city, eastern China: a 15-year population-based survey. BMC Ophthalmol 2018; 18: 159

[13] Chu IY, Alam P, Larson HJ, et al. Social consequences of mass quarantine during epidemics: a systematic review with implications for the COVID-19 response. J Travel Med 2020; 27: taaa192

[14] Cohen AK, Rai M, Rehkopf DH, et al. Educational attainment and obesity: A systematic review. Obes Rev 2013; 14: 989-1005

[15] Cohen S. Psychosocial Vulnerabilities to Upper Respiratory Infectious Illness: Implications for Susceptibility to Coronavirus Disease 2019 (COVID-19). Perspectives on Psychological Science 2021; 16: $161-174$

[16] Cooper J, Tkatchenko AV. A Review of Current Concepts of the Etiology and Treatment of Myopia. Eye \& Contact Lens 2018; 44: 231-247

[17] Crits-Christoph A, et al. Genome sequencing of sewage detects regionally prevalent SARS-CoV-2 variants. mBio 2021; 12:e02703-20

[18] DAK. Mediensucht 2020 - Gaming und Social Media in Zeiten von Corona. DAK-Längsschnittstudie: Befragung von Kindern, Jugendlichen (12-17 Jahre) und deren Eltern https://www.dak.de/dak/ meine-familie/mediensucht-bei-kindern-2295590.html\#|; abgerufen am 30.7.2020

[19] De Witte M. Instead of social distancing, practice 'distant socializing' instead, urges Stanford psychologist. Stanford News, March 19th, 2020 https://news.stanford.edu/2020/03/19/try-distant-socializing-instead/; accessed February 2nd 2021

[20] Ding D, Lawson KD, Kolbe-Alexander TL, et al. The economic burden of physical inactivity: a global analysis of major non-communicable diseases. Lancet 2016; 388: 1311-1324

[21] Engzell P, Frey A, Verhagen M. Learning inequality during the COVID-19 pandemic. 2020; 1-45 doi:10.31235/osf.io/ve4z7

[22] Enthoven CA, Tideman JWL, Polling JR, et al. The impact of computer use on myopia development in childhood: The Generation R study. Prev Med 2020; 132: 105988

[23] Eyimaya OA, Irmak AY. Relationship between parenting practices and children's screen time during the COVID-19 Pandemic in Turkey. J Pediatr Nurs 2020; 56: 24-29

[24] Fernandez-Montero A, et al. The impact of computer use in myopia progression: a cohort study in Spain. Prev Med 2015; 71: 67-71

[25] Flitcroft DI. The complex interactions of retinal, optical and environmental factors in myopia aetiology. Prog Retin Eye Res 2015; 31: 622-660

[26] Gillmann B, Anger H, Specht F. Der Schulausfall in der Pandemie könnte 3,3 Billionen Euro kosten. Handelsblatt 2021 https:// www.handelsblatt.com/politik/deutschland/bildung-und-einkommen-der-schulausfall-in-der-pandemie-koennte-3-3-billionen-euro-kosten/26786872.html; abgerufen am 21.1.2021

[27] Golberstein E, Wen H, Miller BF. Coronavirus Disease 2019 (COVID-19) and Mental Health for Children and Adolescents. JAMA Pediatrics 2020; 174: 819-820

[28] Gold JAW, Rossen LM, Ahmad FB, et al. Race, ethnicity, and age trends in persons who died from COVID-19-United States, May-August 2020. MMWR Morb Mortal Wkly Rep 2020; 69: 1517-1521

[29] Goldstein E, Lipsitch M, Cevik M. On the Effect of Age on the Transmission of SARS-CoV-2 in Households, Schools, and the Community. J Infect Dis 2021; 223: 362-369
[30] Grill M. Wann könnten Schulen wieder öffnen? Tagesschau, Faktenfinder vom 4.2.2021, 8.15 Uhr https://www.tagesschau.de/faktenfinder/ schulen-studien-101.html; abgerufen am 4.2.2021

[31] Gudbjartsson DF, Helgason A, Jonsson H, et al. Spread of SARS-CoV-2 in the Icelandic Population. N Engl J Med 2020; 382: 2302-2315

[32] Hancox RJ, Milne BJ, Poulton R. Association between child and adolescent television viewing and adult health: a longitudinal birth cohort study. Lancet 2004; 364: 257 - 262

[33] Hansen $\mathrm{MH}$, et al. Low physical activity and higher use of screen devices are associated with myopia at the age of $16-17$ years in the CCC2000 Eye Study. Acta Ophthalmol 2020; 98: 315-321

[34] Harrington SC, Stack J, O'Dwyer V. Risk factors associated with myopia in schoolchildren in Ireland. Br J Ophthalmol 2019; 103 : 1803-1809

[35] Hart OE, Rolf U, Halden RU. Computational analysis of SARS-CoV-2/ COVID-19 surveillance by wastewater-based epidemiology locally and globally: Feasibility,economy, opportunities and challenges. Science of the Total Environment 2020; 730: 138875

[36] Ho C-L, Wu W-F, Liou YM. Dose-Response Relationship of Outdoor Exposure and Myopia Indicators: A Systematic Review and Meta-Analysis of Various Research Methods. Int J Environ Res Public Health 2019; 16: 2595

[37] Hoch M, Vogel S, Kolberg L, et al. Weekly SARS-CoV-2 sentinel in primary schools, kindergartens and nurseries, June to November 2020, Germany. medRxiv 2021. doi: https://doi. org/10.1101/2021.01.22.21249971

[38] Holden BA, Fricke TR, Wilson DA, et al. Global Prevalence of Myopia and High Myopia and Temporal Trends from 2000 through 2050. Ophthalmology 2016; 123: 1036-1042

[39] Holden BA, Sankaridurg P, Smith E, et al. Myopia, an underrated global challenge to vision: where the current data takes us on myopia control. Eye 2014; 28: 142-146

[40] Holt-Lunstad J. A pandemic of social isolation? World Psychiatry 2021; 20: 55-56

[41] Holt-Lunstad J, Smith TB, Baker M, et al. Loneliness and social isolation as risk factors for mortality: A meta-analytic review. Perspectives on Psychological Science 2015; 10: 227-237

[42] Honein MA, Barrios LC, Brooks JT. Data and Policy to Guide Opening Schools Safely to Limit the Spread of SARS-CoV-2 Infection. JAMA 2021. doi:10.1001/jama.2021.0374

[43] Hopf S, Pfeiffer N. Epidemiology of myopia. [Article in German] Ophthalmologe 2017; 114: 20-23

[44] Hyde Z. COVID-19, children and schools: overlooked and at risk. Med J Aust 2020; 213: 444-446.e1

[45] Jahn J, Dreifuss D, Topolsky I, et al. Detection of SARS-CoV-2 variants in Switzerland by genomic analysis of wastewater samples. medRxiv 2021. doi.org/10.1101/2021.01.08.21249379

[46] Jiao WY, et al. Behavioral and Emotional Disorders in Children during the COVID-19 Epidemic. J Pediatr 2020; 221: 264-266

[47] JIM. Medienpädagogischer Verbund Südwest: Studie Jugend, Information, Medien (JIM) 2019. Basisuntersuchung zum Medienumgang 12- bis 19-Jähriger (with English summary) Stuttgart 2019

[48] Jung SK, et al. Prevalence of myopia and its association with body stature and educational level in 19-year-old male conscripts in seoul, South Korea. Invest Ophthalmol 2019; 53: 5579-5583

[49] Khan MA, Moverley Smith JE. "Covibesity," a new pandemic. Obes Med 2020; 19: 100282

[50] Kim L, Whitaker M, O'Halloran A, et al. Hospitalization rates and characteristics of children aged $<18$ years hospitalized with laboratory-confirmed COVID-19-COVID-NET, 14 states, March 1-July 25, 2020. MMWR Morb Mortal Wkly Rep 2020; 69: 1081-1088

[51] Klaver CCW, Polling JR, Enthoven CA. 2020 as the Year of Quarantine Myopia. JAMA Ophthalmology, published online January 2021; 14, 2021, E1-E2 
[52] Kohl HW, Craig CL, Lambert EV, et al. The pandemic of physical inactivity: global action for public health. Lancet 2012; 380: 294-305

[53] Kuhl PK. Brain mechanisms in early language acquisition. Neuron 2010; 67: 713-727

[54] Kuhl PK, Tsao FM, Liu HM. Foreign-language experience in infancy: effects of short-term exposure and social interaction on phonetic learning. PNAS 2003; 100: 9096-9101

[55] Lavezzo E, et al. Suppression of a SARS-CoV-2 outbreak in the Italian municipality of Vo'. Nature 2020; 584: 425-429

[56] Lawton G. New coronavirus variants detected in sewage before showing up in tests. New Scientist 2021; 3319 https://www.newscientist.com/article/mg24933193-200-new-coronavirus-variants-detected-in-sewage-before-showing-up-in-tests/\#ixzz6krwa0NxO; abgerufen am 28.1.2021

[57] Lee I-M, et al. Impact of Physical Inactivity on the World's Major Non-Communicable Diseases. Lancet 2012; 380: 219-229

[58] Lillard AS, Peterson J. The immediate impact of different types of television on young children's executive function. Pediatrics 2011; 128: 649-655

[59] Loades ME, Chatburn E, Higson-Sweeney N, et al. Rapid Systematic Review: The Impact of Social Isolation and Loneliness on the Mental Health of Children and Adolescents in the Context of COVID-19. J Am Acad Child Adolesc Psychiatry 2020; 59: 1218-1239

[60] López-Bueno R, López-Sánchez GF, Casajús JA, et al. Potential health-related behaviors for pre-school and school-aged children during COVID-19 lockdown: A narrative review. Prev Med 2021; 143: 106349

[61] Lu T. Almost half of smartphone users spend more than 5 hours a day on their mobile device. Counterpoint Research, October 12th, 2017 https://www.counterpointresearch.com/almost-half-of-smartphone-users-spend-more-than-5-hours-a-day-on-their-mobile-device/; accessed January 6th, 2018)

[62] Ludvigsson JF, Engerström L, Nordenhäll C, et al. Open schools, Covid-19, and child and teacher morbidity in Sweden. N Engl J Med 2021. doi: 10.1056/NEJMc2026670

[63] Madewell Z], Yang Y, Longini IM et al. Household Transmission of SARS-CoV-2: A Systematic Review and Meta-analysis. JAMA Netw Open 2020; 3(12): e2031756

[64] McIntyre RS, Lee Y. Projected increases in suicide in Canada as a consequence of COVID-19. Psychiatry Research 2020; 290: 113104

[65] Meidinger H-P. Lehrerverbandschef befürchtet wieder SchulschlieBungen. Frankenpost 21.3.2021 www.frankenpost.de/inhalt. deutschland-lehrerverbandschef-befuerchtet-wieder-schulschliessungen.85e7f421-2ff0-4578-a3d2-b6eb86ae9d04.html; abgerufen am 23.3.2021

[66] Melvin RG, et al. Predictive power of SARS-CoV-2 wastewater surveillance for diverse populations across a large geographical range. medRxiv 2021 doi: 10.1101/2021.01.23.21250376

[67] Mirshahi A, PontoKA, HoehnRetal. Myopia and level of education: results from the Gutenberg Health Study. Ophthalmology 2014; 121: 2047-2052

[68] Moore SA, et al. Impact of the COVID-19 virus outbreak on movement and play behaviours of Canadian children and youth: a national survey. Int J Behav Nutr Phys Act 2020; 17(1): 85

[69] Morgan IG, Ohno-Matsui K, Saw SM. Myopia. Lancet 2012; 379: 1739-1748

[70] Morgan IG, Rose KA. Myopia and international educational performance. Ophthalmic Physiol Opt 2013; 33: 329-338

[71] Mountjoy E, et al. Education and myopia: assessing the direction of causality by mendelian randomisation BMJ 2018; 361: k2022

[72] Nickels S, Hopf S, Pfeiffer N, et al. Myopia is associated with education: Results from NHANES 1999-2008. PLoS One 2019; 14: e0211196

[73] Nikkelen SW, Valkenburg PM, Huizinga M, et al. Media use and ADHDrelated behaviors in children and adolescents: A meta-analysis. Dev Psychol 2014; 50: 2228-2241
[74] O'Leary ST. To Spread or Not to Spread SARS-CoV-2-Is That the Question? JAMA Pediatr 2021. doi:10.1001/jamapediatrics.2021.0006

[75] Oster E. Schools are not spreading covid-19. This new data makes the case. Washington Post 20.11.2020 https://www.washingtonpost. com/opinions/2020/11/20/covid-19-schools-data-reopening-safety/?arc404=true; abgerufen am 22.11.2020

[76] Padrón I, Fraga I, Vieitez L, et al. A Study on the Psychological Wound of COVID-19 in University Students. Front Psychol 2021; 12: 56887

[77] Park M, et al. The impact of childhood obesity on morbidity and mortality in adulthood: a systematic review. Obes Rev 2012; 985-1000

[78] Peiser ]. The University of Arizona says it caught a dorm's covid-19 outbreak before it started. The Washington Post 28.8.2020 https:// www.washingtonpost.com/nation/2020/08/28/arizona-coronavirus-wastewater-testing/; abgerufen am 29.8.2020

[79] Pew Research Center. Smartphone Ownership and Internet Usage Continues to Climb in Emerging Economies, February, 2016 www. pewglobal.org/files/2016/02/pew_research_center_global_technology_report_final_february_22__2016.pdf; accessed Jan 6th 2018

[80] Pietrobelli A, Pecoraro L, Ferruzzi A, et al. Effects of COVID-19 Lockdown on Lifestyle Behaviors in Children with Obesity Living in Verona, Italy: A Longitudinal Study. Obesity 2020; 28: 1382-1385

[81] Plotnikov D, et al. Effect of Education on Myopia: Evidence from the United Kingdom ROSLA 1972 Reform. Invest Ophthalmol Vis Sci 2020; 61: 7

[82] Polanczyk GV, et al. Annual research review: a meta-analysis of the worldwide prevalence of mental disorders in children and adolescents. J Child Psychol Psychiatry 2015; 56: 345-365

[83] Poletti M. Hey teachers! Do not leave them kids alone! Envisioning schools during and after the coronavirus (COVID-19) pandemic. Trends in Neuroscience and Education 2020; 20: 100140

[84] Pollán M, Pérez-Gómez B, Pastor-Barriuso R, et al. Prevalence of SARS-CoV-2 in Spain (ENE-COVID): a nationwide, population-based seroepidemiological study. Lancet 2020; 396: 535-544

[85] Pombo A, Luz C, Rodrigues LP, et al. COVID-19 confinement in Portugal: effects on the household routines of children under 13. Research Square 2020. doi.org/10.21203/rs.3.rs-45764/v1

[86] Public Health England. Investigation of novel SARS-CoV-2 variant. Variant of Concern 202012/01. Technical briefing 5 https://assets. publishing.service.gov.uk/government/uploads/system/uploads/attachment_data/file/957504/Variant_of_Concern_VOC_202012_01_ Technical_Briefing_5_England.pdf; abgerufen am 3.2.2021

[87] Ravens-Sieberer U, Kaman A, Erhart M, et al. Impact of the COVID-19 pandemic on quality of life and mental health in children and adolescents in Germany. Eur Child Adolesc Psychiatry 2021. doi. org/10.1007/s00787-021-01726-5

[88] Richards R, et al. Adolescent screen time and attachment to parents and peers. Arch Pediatr Adolesc Med 2011; 164: 258-262

[89] Roberto CA, Swinburn B, Hawkes C, et al. Patchy progress on obesity prevention: emerging examples, entrenched barriers, and new thinking. Lancet 2018; 385: 2400-2409

[90] Robertson LA, McAnally HM, Hancox RJ. Childhood and adolescent television viewing and antisocial behavior in early adulthood. Pediatrics 2013; 131: 439-446

[91] Robinson TN, Banda JA, Hale L, et al. Screen media exposure and obesity in children and adolescents. Pediatrics 2017; 140: S97

[92] Rudnicka A, Kepatranakis VV, Wathern AK, et al. Global variations and time trends in the prevalence of childhood myopia, a systematic review and quantitative meta-analysis: implications for aetiology and early prevention. Br J Ophthalmol 2016; 100: 882-890

[93] Schaeffel F. Biological mechanisms of myopia [Article in German]. Ophthalmologe 2017; 114: 5-19

[94] Schaeffel F, Glasser A, Howland HC. Accommodation, refractive error and eye growth in chickens. Vision Res 1988; 28: 639-657

[95] Simon J, Helter TM, White RG, et al. Impacts of the Covid-19 lockdown and relevant vulnerabilities on capability well-being, mental 
health and social support: an Austrian survey study. BMC Public Health 2021; 21: 314

[96] Singh S, Roy D, Sinha K, et al. Impact of COVID-19 and lockdown on mental health of children and adolescents: A narrative review with recommendations. Psychiatry Res 2020; 293: 113429

[97] Spitzer M. The Smartphone Epidemic. Risks for Health, Education, and Society [Title in German]. Stuttgart: Klett, 2018

[98] Spitzer M. Loneliness [Title in German]. München: Droemer, 2018

[99] Spitzer M. Pandemic. What corona does to us and what we are making out of it [Title in German]. München: MVG, 2020

[100] Spitzer M. Masked education? The benefits and burdens of wearing face masks in schools during the current Corona pandemic. Trends in Neuroscience and Education 2020; 20: 100138

[101] Spitzer M. Mediennutzung in Zeiten von Corona. Nervenheilkunde 2020; 39: 698-703

[102] Spitzer M. Wen sollen wir Impfen. Nervenheilkunde 2021: 40: 367-376

[103] Spitzer M. Naturerleben. Stuttgart: Klett, 2021

[104] Streb J, Kammer T, Spitzer M, et al. Extremely reduced motion in front of screens: Investigating real-world physical activity of adolescents by accelerometry and electronic diary. PLoS ONE 2015; 10: e0 126722

[105] Stringhini S, Wisniak A, Piumatti G, et al. Seroprevalence of antiSARS-CoV-2 IgG antibodies in Geneva, Switzerland (SEROCoV-POP): a population-based study. Lancet 2005; 396: 313-319

[106] Thomasius R. Kernergebnisse der Studie in Grafiken. Gaming und Social-Media - Das Nutzungsverhalten 10- bis 17-Jähriger und ihrer Eltern vor und unter dem Corona-Lockdown. In: Mediensucht 2020 - Gaming und Social Media in Zeiten von Corona. DAK-Längsschnittstudie: Befragung von Kindern, Jugendlichen (12-17 Jahre) und deren Eltern. Juli 2020. DAK Gesundheit 2020, 80-86

[107] Thomée S, Lissner L, Hagberg M, et al. Leisure time computer use and overweight development in young adults - a prospective study. BMC Public Health 2015; 15: 839

[108] Tideman JW, et al. Myopia, a growing health problem [Article in Dutch]. Ned Tijdschr Geneeskd 2016; 160: D803

[109] Tönshoff B, et al. Prevalence of SARS-CoV-2 Infection in Children and Their Parents in Southwest Germany. JAMA Pediatr 2021; e210001

[110] Torales J, O'Higgins M, Castaldelli-Maia JM, et al. The outbreak of COVID-19 coronavirus and its impact on globalmental health. Int J Soc Psychiatry 2020; 66: 317-320

[111] Tremmel M, Gerdtham U-G, Nilsson PM, et al. Economic Burden of Obesity: A Systematic Literature Review. Int J Environ Res Public Health 2017; 14: 435

[112] UNESCO. Education: From disruption to recovery. School clusures caused by Coronavirus (Covid-19). https://en.unesco.org/covid19/ educationresponse, accessed July 11 th 2020

[113] Van Lancker W, Parolin Z. COVID-19, school closures, and child poverty: a social crisis in the making. Lancet 2020; e243-e244

[114] Vitale S, et al. Prevalence of Refractive Error in the United States, 1999-2004. Arch Ophthalmol 2008; 126: 1111-1119

[115] Vogel G. How Sweden wasted a 'rare opportunity' to study coronavirus in schools. Science 2020. doi:10.1126/science.abc9565

[116] Vogel G. Data in paper about Swedish schoolchildren come under fire. Science 2021; 371: 973-974
[117] Von Zehender CW. Über den Einfluss des Schul-Unterrichts auf Entstehung von Kurzsichtigkeit. Vortrag auf Veranlassung des Vereins-Vorstandes für öffentliche Gesundheitspflege in Rostock am 25. Februar 1880. Nebst einem Anhang enthaltend Entgegnungen von fünfundzwanzig Lehrern des Rostocker Gymnasiums und der Realschule. Stuttgart: Ferdinand von Enke, 1880

[118] Wallman J, Winawer J. Homeostasis of eye growth and the question of myopia. Neuron 2004; 43: 447-68

[119] Wang J, Li M, Zhu D, et al. Smartphone Overuse and Visual Impairment in Children and Young Adults: Systematic Review and Meta-Analysis. J Med Internet Res 2020; 22

[120] Wang J, Li Y, Musch DC, et al. Progression of Myopia in School-Aged Children After COVID-19 Home Confinement. JAMA Ophthalmology 2021; e206239

[121] Warner N. Update on myopia. Curr Opin Ophthalmol 2016; 27 : 402-406

[122] Wen C P, Wu X. Stressing harms of physical inactivity to promote exercise. Lancet 2012; 380: 192-193

[123] Williams KM, Bertelsen G, Cumberland P, et al. European Eye Epidemiology ( $E(3))$ Consortium. Increasing Prevalence of Myopia in Europe and the Impact of Education. Ophthalmology 2015; 122: 1489-1497

[124] Williams KM, Kraphol E, Yonova-Doing E, et al. Early life factors for myopia in the British Twins Early Development Study. Br J Ophthalmol 2019; 103: 1078-1084

[125] Williams KM, Verhoefen VJM, Cumberland P et al. Prevalence of refractive error in Europe: the European Eye Epidemiology (E3) Consortium. Eur J Epidemiol 2015; 30: 305-315

[126] Wößmann L. Folgekosten ausbleibenden Lernens: Was wir über die Corona-bedingten Schulschließungen aus der Forschung lernen können. Ifo Schnelldienst 2020; 73: 38-44

[127] Wu JF, Bi HS, Wang SM, et al. Refractive error, visual acuity and causes of vision loss in children in Shandong, China. The Shandong Children Eye Study. PLoS ONE 2013; 8(12): e82763

[128] Wu KJ. Massachusetts actually might have a way to keep schools open. The Atlantik, 4.3.2021

[129] Xie X, Xue Q, Zhou Y, et al. Mental Health Status Among Children in Home Confinement During the Coronavirus Disease 2019 Outbreak in Hubei Province, China. JAMA Pediatr 2020; 174: 898-900

[130] Zhang L, Wang W, Dong X, et al. Association between time spent outdoors and myopia among junior high school students: A 3-wave panel study in China. Medicine 2020; 99: e23462

[131] Zhao Y, Guo Y, Xiao Y, et al. The effects of online homeschooling on children, parents, and teachers of grades 1-9 during the COVID-19 pandemic. Med Sci Monit 2020; 26: e925591

[132] Zhou S], et al. Prevalence and socio-demographic correlates of psychological health problems in Chinese adolescents during the outbreak of COVID-19. Eur Child Adolesc Psychiatry 2020; 29: 749-758

[133] Koopmann A, Georgiadou E, Kiefer F, et al. Did the General Population in Germany Drink More Alcohol during the COVID-19 Pandemic Lockdown? Alcohol and Alcoholism 2020; 55: 698-699

[134] Groshkova T, Stoian T, Cunningham A, et al. Will the Current COVID-19 Pandemic Impact on Long-term Cannabis Buying Practices?. J Addict Med 2020; 14: 10.1097 